\title{
Desarrollo de competencias profesionales en biomedicina utilizando el aprendizaje basado en proyectos
}

\author{
Isaias Sanmartin Santos ${ }^{a}$, Ana Lloret Alcañiz ${ }^{b}$, Jesús Ángel Prieto Ruiz, Ignacio Ventura González ${ }^{c}$ \\ a. Departamento de Ciencias Médicas Básicas. Facultad de Veterinaria y Ciencias Experimentales. Universidad \\ Católica de Valencia San Vicente Mártir. \\ b. Departamento de Fisiología. Facultad de Medicina. Universidad de Valencia. \\ c. Departamento de Ciencias Médicas Básicas. Facultad de Medicina. Universidad Católica de Valencia San \\ Vicente Mártir.
}

\begin{abstract}
Las competencias profesionales en el ámbito de las ciencias experimentales se adquieren con la práctica del ejercicio profesional. En el ámbito universitario, se imparten de forma parcelada los conocimientos, destrezas y habilidades en las diferentes asignaturas de grado. Nuestros 45 alumnos del grado de Biotecnología de la Universidad Católica de Valencia, durante los periodos de prácticas repiten protocolos y siguen una receta para realizar un experimento que ejemplarice los contenidos fundamentales de la asignatura que se está impartiendo. El abordaje de este trabajo se basa en el aprendizaje basado en proyectos. El alumno ha de elaborar un proyecto biotecnológico de principio a fin. El proyecto se basa en la construcción de un vector no vírico para inmunoterapia, que va desde la temporalización de los experimentos hasta la elaboración del artículo que debería representar el resultado de su aprendizaje. El alumno, ha de elaborar sus protocolos, calcular los tiempos, sincronizar los cultivos y usar diferentes técnicas aprendidas en cursos anteriores. Esta experiencia próxima a la realidad futura le obliga a trabajar las competencias de diversas asignaturas del grado, no solo inmunología. Las ventajas que ofrece este tipo de trabajo es aumentar la autonomía en el laboratorio y poner en práctica lo aprendido durante el grado para que le sea significativo su aprendizaje.
\end{abstract}

Palabras clave: competencias profesionales, aprendizaje basado en proyectos.

\begin{abstract}
Professional competences in the field of experimental sciences are acquired through the practice of professional practice. In the university environment, the knowledge, skills and abilities in the different undergraduate subjects are taught in a parceled manner. Our 45 students biotechnology studies in Catholic University of Valencia, during the internship repeat protolocos and follow a recipe to perform an experiment that exemplifies the fundamental contents of the subject being taught. The aboradaje of this work is based on
\end{abstract}


project-based learning. The student has to elaborate a biotechnological project from beginning to end. The project is based on the construction of a non-viral vector for immunotherapy, which ranges from the timing of the experiments to the preparation of the article that should represent the result of their learning. The student has to elaborate his protocols, calculate the times, synchronize the crops and use different techniques learned in previous courses. This experience, close to the future reality, forces him to work on the competences of diverse asignturas of the degree, not only immunology. The advantages offered by this type of work is to increase the autonomy in the laboratory and put into practice what was learned during the degree so that their learning is meaningful.

Keywords: immunology, project-learning, immunization, cancer, vaccine, non-viral vector, lipoplex,

\section{Introducción}

Las competencias profesionales en biotecnología son a menudo un aspecto olvidado de la formación que ofrecemos a nuestros alumnos. Las distintas asignaturas que componen el Grado se enfocan al desarrollo conceptual del cuerpo de conocimientos específico de la materia que tratan, olvidando un aspecto fundamental: el futuro egresado se someterá a desafíos en su puesto laboral, desafíos directamente relacionados con la aplicación práctica, creativa y coordinada de los conocimientos teóricos que aprendió en sus estudios. Sin embargo, en la actualidad ninguna asignatura de nuestra titulación (Biotecnología), más allá del trabajo final de grado (TFG), ofrece un marco pedagógico específico para el desarrollo de estas competencias. En particular, la "capacidad de aplicar los conocimientos en la práctica", las "habilidades de investigación", "analizar e interpretar datos cientificos en el ámbito de la Biotecnología", "resolver y analizar problemas derivados de la práctica de la biotecnología" y "describir y medir variables relevantes de procesos o experimentos" son fundamentales para la práctica profesional. También, la capacidad para trabajar en un equipo de I\&D.

A pesar de ello, existe en la universidad una escasez de enfoque en la educación que forme eficazmente con las competencias que demandan el mercado laboral y las empresas.

El Aprendizaje Basado en Proyectos (PBL, Project-based learning) es una metodología eficaz para desarrollarlas (Markham, 2003). Organizados en grupos, los alumnos completan un proyecto diseñado por el profesor, a lo largo del cual son expuestos a similares retos y situaciones complejas que se dan en el ámbito profesional real. La interactividad entre los miembros del grupo al enfrentarse a los problemas a resolver en el transcurso del proyecto, favorece aprendizajes valiosos para adquirir las competencias del trabajo en equipo. Los métodos y conocimientos técnicos que deben aplicar tienen relación directa con los adquiridos durante los cursos anteriores del Grado, de manera que el proyecto ofrece una aplicación práctica integradora de diversas competencias desarrolladas durante los estudios. Y por último, el Aprendizaje Basado en Proyectos resulta en sí mismo muy motivador. 
Resulta necesario además tener en cuenta una serie de aspectos que resultan claves para el éxito de la actividad PBL (Valero-García, 2011). Aunque el proyecto sea complejo, debe ser percibido por los estudiantes como asequible, y debe planificarse cuidadosamente presentando un plan de trabajo o cronograma para que pueda llegarse al objetivo previsto en el escaso tiempo disponible en las sesiones prácticas. Diseñar un buen método de evaluación es también una clave importante, así cómo generar la percepción en los estudiantes de que deben desarrollar un trabajo en equipo eficaz si quieren alcanzar la consecución del proyecto en el tiempo previsto. El trabajo desarrollado ha de ser monitorizado por el profesor mediante una serie de entregas de resultados parciales, de manera que no se confíe la supervisión de todo el trabajo a una única entrega final en la que ya no pueden corregirse los errores o asesorar a los estudiantes.

En el presente trabajo, además, hermos seleccionado particularmente el proyecto escogido para aumentar la motivación en nuestros alumnos. Deben construir desde el inicio un vehículo de transferencia de antígenos para inmunicación antitumoral, que pueden "visualizar" al final de sus prácticas a través del microscopio (Sanmartín, 2010). La motivación es un factor muy importante para generar la actitud adecuada que permita concluir con éxito el proyecto, ya que es de envergadura profesional y no resulta tan fácil o directo como las prácticas de laboratorio del estilo "seguir el protocolo del cuadernillo de prácticas" al que los alumnos están acostumbrados.

Nuestro propósito es diseñar un proyecto biotecnológico de nivel profesional a realizar por los propios alumnos, que pueda desarrollarse a lo largo de las únicas cinco sesiones disponibles de prácticas de laboratorio de la asignatura "Inmunología para biotecnología", y dirigido a desarrollar específicamente las competencias anteriormente citadas.

En lugar de seguir protocolos escritos en un cuadernillo de prácticas para demostrar algunas técnicas de laboratorio independientes y no relacionadas entre sí, como es habitual, los alumnos reciben la siguiente propuesta del profesor: "formáis parte del equipo de $I \& D$ de una empresa de biomedicina especializada en nanotecnología del cáncer, y se os pide que diseñéis, construyáis y evaluéis un vector basado en antígenos proteicos, liposomas y ADN para inmunización antitumoral".

Los alumnos se organizarán en grupos o equipos, simulando el equipo de desarrollo de un producto biotecnológico, y utilizarán técnicas y métodos en su mayoría ya aprendidos en los cursos anteriores del Grado.

En resumen, en el presente trabajo describimos cómo hemos utilizado la metodología de aprendizaje basado en proyectos para desarrollar competencias profesionales que encajan con las que demanda el mercado laboral, tan necesarias pero a menudo olvidadas en los estudios técnico-científicos. Esperamos que a través de este nuestros alumnos egresados desarrollen la necesaria confianza en sí mismos que les permita utilizar los conocimientos adquiridos en el Grado para ser competentes y abordar con éxito sus futuros proyectos a nivel profesional.

Para la consecución del proyecto, se deben desarrollar los siguientes objetivos parciales, para asegurarnos de que los alumnos poseen toda la información y materiales para abordarlo con éxito. 


\section{Objetivos:}

Los objetivos del trabajo se han divido en dos partes: Los objetivos generales correspondientes al trabajo experimental de laboratorio y los objetivos del proyecto de innovación docente. En primer lugar, citaremos los objetivos experimentales que los alumnos deben de alcanzar para representar el currículo de las diferentes áreas de ciencias experimentales dentro del grado de biotecnología.

Construcción "de novo" del vector de inmunización antitumoral (LPP).

1. Validación experimental y estudios dosis-respuesta de los vectores de ADN basados en lipopoliplejos (LPP).

2. Elaboración de un artículo científico donde trabajen las competencias propias de las ciencias experimentales del grado de biotecnología.

3. Auto-evaluación del proceso de investigación.

\section{Objetivos Experimentales}

1. Obtención del vector de inmunización.

1. Extracción del plásmido de ADN de bacterias transformadas

2. Extracción de antígenos proteicos de células HeLa

3. Preparación de liposomas.

4. Cuantificación de los productos anteriores mediante las técnicas habituales (Bradford ${ }^{\circledR}$ y cuantificación del ADN por espectrofotometría).

5. Preparación de liposomas mediante la encapsulación de proteínas antigénicas procedentes de la membrana de las células HeLa y la cuantificación del rendimiento de encapsulación.

6. Preparación de los complejos ADN/PEI.

7. Construcción del vector LPP.

8. Ensayo Cesio/carboxifluoresceina para determinar la ratio poliplejos/liposomas

2. Caracterización de los diferentes LPP obtenidos, calculando las relaciones lípido/proteína para la optimización del complejo.

3. Desarrollo de un ensayo en células HeLa para estudiar la dosis-respuesta del vector antitumoral.

1. Mantenimiento y sincronización de cultivos celulares.

2. Cuantificación de muerte celular.

\section{Objetivos pedagógicos}

1. Fomentar autonomía personal y profesional en el ámbito de las ciencias experimentales en el grado de biotecnología mediante un aprendizaje cooperativo.

2. Favorecer un aprendizaje significativo basado en una experiencia real de diseño de un proyecto de investigación para la elaboración de una vacuna antitumoral en una empresa biotecnológica.

\section{Desarrollo de la innovación}

Se trata de un proyecto complejo y de envergadura profesional, y que trata de múltiples técnicas y aspectos muy interesantes y motivadores para la formación de nuestros alumnos, 
pero que deben adaptarse para que sean asequibles. Y estructurarse de forma adecuada y sistemática para constituir una verdadera actividad de "aprendizaje basado en proyectos".

A partir del análisis y realización del proyecto experimental profesional por los profesores, se pulirán los aspectos necesarios y se procederá a determinar cuales de estas técnicas y métodos son los más apropiados para ser elaborados en su totalidad por los alumnos. Y, asimismo, cuáles pueden ser abreviados o aligerados suministrando los materiales parciales preparados por el profesor. También será necesario determinar los pasos experimentales que requerirán más ayuda, y el desarrollo de preguntas o cuestiones desarrolladas específicamente para el proyecto. En resumen, todos los aspectos necesarios para convertir lo que es un proyecto "profesional" en una verdadera innovación a nivel de prácticas de laboratorio bajo la metodología "aprendizaje basado en proyectos" para nuestros alumnos.

El desarrollo de la innovación consiste en la integración de conocimientos prácticos de diferentes áreas de conocimiento del grado de biotecnología, así como la distribución del tiempo de laboratorio, tan relevante en el trabajo científico. Basándonos en la metodología de aprendizaje basado en proyectos se ha planteado en primer lugar; elaboración de un cronograma donde el alumno ha de ser capaz de distribuir los experimentos en el tiempo para así, poder sincronizar los cultivos celulares y obtención de los diferentes compuestos. En segundo lugar, recordar las prácticas de cursos anteriores, tales como; Biología Celular y Cultivos celulares; Química Orgánica para la elaboración del lipopoliplejo; Bioquímica I y II para la valoración por espectrofotometría de complejos de DNA/lípidos; y evidentemente inmunología, tal y como se muestra en la siguiente tabla.

\section{$\begin{array}{llll}\text { Asignatura } & \text { Curso } & \text { Fase del proyecto } & \text { Ej. Experimentos }\end{array}$}

\begin{tabular}{lccc}
\hline Biología Celular & 1 & Preparativo & Recuento de viables \\
Bioquímica I y II & 1 y 2 & Preparativo & Cuantificación lipopoliplejo \\
Química Orgánica & 1 & Preparativa & Elaboración del complejo \\
Cultivos Celulares & 3 & Preparativa & Cultivo de macrófagos \\
Inmunología & 4 & Interpretación de resultados & Conclusiones
\end{tabular}

Tabla 1. Grado en Biotecnología y materias integradas en el proyecto de inmunología.

Para la elaboración del cronograma el alumno ha de tener en cuenta varias variables en el tiempo, tales como el crecimiento de los macrófagos y de las células HeLa. Dicha temporalización es compleja hasta para los profesionales que ejercen diariamente en laboratorios de biotecnología, ya que requiere elaborar un eje temporal de los experimentos para tener las células en optimas condiciones y perfectamente sincronizadas. Precisamente, este ejercicio ha sido el más complejo a la hora de realizar la tarea. Los profesores implicados en el proyecto hemos tenido que sugerir una temporalización de los experimentos. A los 
Desarrollo de competencias profesionales en biomedicina utilizando el aprendizaje basado en proyectos

alumnos se les proporcionó una tabla vacía con las sesiones y los experimentos que debían de realizar y ellos situaban los experimentos en los horarios, tal y como figura en la tabla 2.

\begin{tabular}{|c|c|c|c|c|c|c|c|}
\hline $\begin{array}{c}\text { Sesión 1 } \\
\text { Lab. }\end{array}$ & Sesión 2 & Sesión 3 & Sesión 4 & Sesión 5 & Sesión 6 & Aula 1 & Aula 2 \\
Lab. & Lab. & Lab. & Lab. & Lab. & Previa & Final \\
\hline Obj. Exp. 1 & Obj. Exp. 1 & Obj. Exp. 1 & Obj. Exp. 2 & $\begin{array}{c}\text { Obj. Exp. } \\
2\end{array}$ & $\begin{array}{c}\text { Obj. Exp. } \\
3\end{array}$ & $\begin{array}{c}\text { Clase } \\
\text { magistral }\end{array}$ & $\begin{array}{c}\text { Puesta en } \\
\text { Común }\end{array}$ \\
\hline Obj. Exp. 1 & Obj. Exp. 1 & Obj. Exp. 1 & Obj. Exp. 2 & Obj. Exp. & Obj. Exp. & Planificación & Resultados \\
& & & & 2 & 3 & & \\
\hline Lectura/Casa & Lectura/Casa & Tablas/Casa & Tablas/Casa & Datos/Casa & Datos/Casa & Redactar/Casa & Redactar/Casa \\
& & & & & & & \\
\hline
\end{tabular}

Tabla 2. Organización temporal de los experimentos. Las sesiones de laboratorio se dividen en dos partes. Una fase más preparatoria y una fase más experimental y recogida de datos, donde se realizan los objetivos experimentales. Todas las sesiones se acompañan de trabajo en casa y trablajo en el aula/laboratorio. Finalemente, tenemos la puesta en común de los resultados y redacción del proyecto de forma individual.

A los 45 alumnos de $4^{\circ}$ curso de la facultad de Biotecnología de la Universidad Católica de Valencia San Vicente Mártir, se les facilita la lista de los experimentos que deben de realizar y una bibiografía básica, es necesario realizar una sesión previa en el aula para explicar la metodolgía de trabajo, así como también, organizar un plan de trabajo en aula y en casa. Este plan de trabajo individual es importante que el alumno lo cumpla meticulosamente. Si el alumno no es cumplidor no podrá realizar los experimentos, ya que, en las sesiones de laboratorio no hay explicación previa. Este hecho obliga al alumno a prepararse antes de realizar las actividades. Así como también, que la mayor parte de los experimentos requiere de la colaboración de compañeros, aunque no siempre con las mismas parejas, trios etc. Es decir, realmente es un trabajo individual porque todos los alumnos realizan todos los experimentos pero en muchos casos han de colaborar con sus compañeros para determinadas tareas. Por ejemplo, se han de distribuir el tiempo dentro de las dos horas de laboratorio en dos partes ( $1 \mathrm{~h}$ y $2 \mathrm{~h}$ ) debido a que, a diferencia de las prácticas tradicionales, donde el alumno tiene el material sobre su banco. En nuestro caso, existe un banco común donde están todos los reactivos y materiales de uso común. El alumno ha de ir a recoger el material y realizarse sus stocks, etc. En situaciones reales de laboratorios de investigación ocurre lo mismo. Es decir, exite una bancada común y es el investigador el que realiza sus disoluciones, tampones etc. Se ha de distribuir el tiempo y los experimentos para no entrar en conflicto con los demás miembros del departamento. Los profesores hemos participado activamente en este proceso de distribución del tiempo y del espacio ya que es un elemento fundamental del aprendizaje práctico en ciencias experimentales que con el sistema docente de prácticas de laboratorio actual no se tiene en cuenta. 


\section{Resultados}

El complejo de inmunización realizado por los alumnos (figura 1) fue ensamblado y cuantificado adecuadamente, posteriormente transfectado a los macrófagos a una confluencia correcta y finalmente las células inducidas (macrógafos) fueron co-cultivadas con las células tumorales HeLa. Todos estos experimentos integran competencias esenciales del grado.

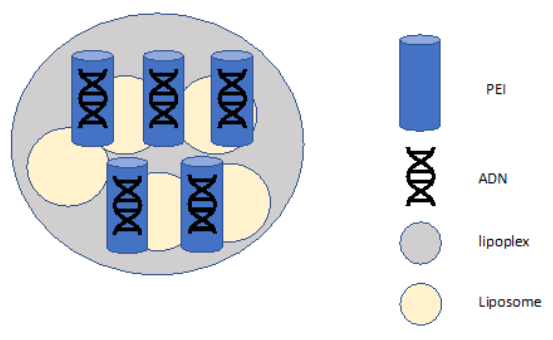

Figura 1. Esquema del complejo lipopoliplejo para la vacunación de células tumorales mediante un vector no viral.

Los resultados de los alumnos se plasmaron en un trabajo con formato "paper" es decir, título, autores con filiación, abstract, palabras clave, introducción, objetivos, resultados, discusión y conclusiones.

Nuestros 45 alumnos de la facultad de Biotecnología de la Universidad Católica de Valencia, realizaron mediante la evaluación del proyecto de innovación docente en dos fases, en primer lugar, un texto libre, respondiendo a la pregunta "¿Qué te han parecido las prácticas de inmunología? Y por otra parte, se realizó una encuesta con la intención de objetivar los resultados. En cuanto al texto libre, los alumnos expresaron su satisfacción por el planteamiento de las prácticas poniendo énfasis en que les había parecido una práctica real. También se desprendió de la encuesta libre que les hubiera gustado tener más tiempo para poder asimilar todos los conocimientos teóricos necesarios para la comprensión de todos los procedimientos. Una vez leídos los textos libres, elaboramos una encuesta de satisfacción donde resumimos los resultados en la siguiente tabla de valoración de 0-4. Siendo 0 muy negativo y 4 muy positivo.

\section{Encuesta de satisfacción}

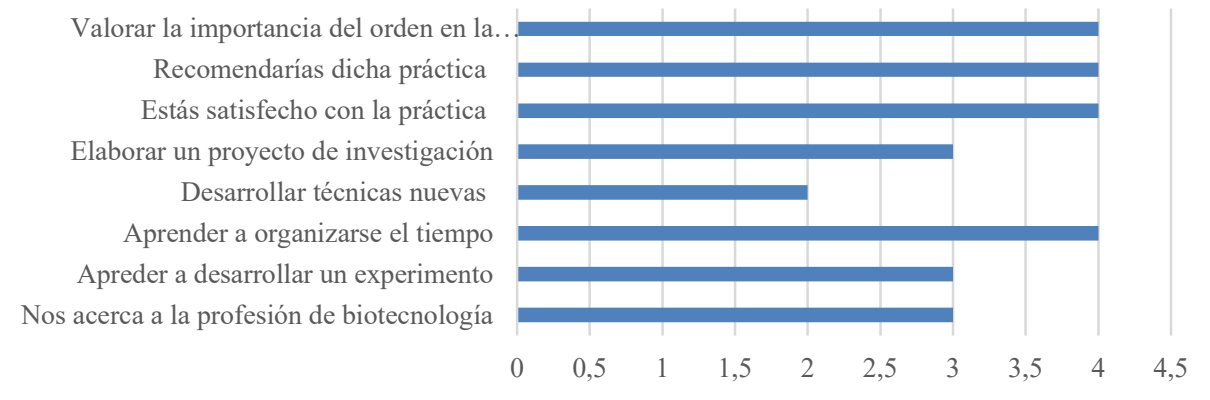

Tabla 3. Resumen de la encuesta de satisfacción de los alumnos que han participado en el proyecto de 
Desarrollo de competencias profesionales en biomedicina utilizando el aprendizaje basado en proyectos

innovación docente de la asignatura de inmunología de 4 año. Particiaparon el 65\% de los alumnos de un total de 45 alumnos.

Una vez terminados los experimentos se valoraron los aspectos negativos:

- Requiere mucho tiempo de elaboración por parte del alumno y del profesor.

- Mayor coste económico por alumno, este tipo de experiencias requiere de material real, no el pseudo-material de prácticas tales como kits de prácticas comerciales, etc.

- Se requieren grupos muy reducidos o laboratorios de alta capacidad, debido a que el alumno no está estático en su puesto. La práctica real de laboratorio requiere de desplazarse por todo el espacio.

- Conocimientos previos afianzados. Los alumnos que no recuerdan conceptos básicos de asignaturas se descuelgan fácilmente de la práctica.

- Dificultades a la hora de realizar los protocolos de forma autónoma. Es necesario ayudarle muchas veces en la organización del tiempo y del material.

Las dificultades que nos enfrentamos fueron subsanadas satisfactoriamente debido a la implicación del profesorado y la buena predisposición de los alumnos, ya que para ellos fue una experiencia innovadora. Según los alumnos nunca habían realizado una experiencia de este tipo durante todo su grado. El grado de satisfacción por parte de los alumnos ha sido muy alto.

\section{Conclusiones}

La realización de un proyecto profesional completo en el último curso del Grado que involucre las habilidades y conocimientos desarrollados por los alumnos a lo largo de sus estudios universitarios es muy conveniente. El uso integrado de los conocimientos adquiridos desarrolla competencias y un sentido de la propia confianza del alumno a la hora de enfrentar retos profesionales.

El trabajo en el laboratorio es fundamental en los grados de ciencias experimentales. Después de la experiencia de este proyecto de innovación docente, donde los estudiantes de inmunología se han acercado a una práctica más real de lo que sería el laboratorio profesional, podemos afirmar que sería conveniente trabajar mediante la metodología ABP en un mayor número de asignatruras de los últimos cursos académicos del Grado en Biotecnología. Por tanto, podemos concluir que:

El aprendizaje basado en proyectos en inmunología, es valorado postivimante por parte de los alumnos para adquirir las competencias profesionales en el grado de biotecnología.

El aprendizaje basado en proyectos ha permitido dar un enfoque globalizador al conocimiento práctico de diferentes materias a lo largo del grado de biotecnología y permite integrarlas para desarrollar un proyecto de investigación por parte de los 45 estudiantes del grado de biotecnología de la Universidad Católica de Valencia San Vicente Mártir.

\section{Referencias}

(c)) EY-NC-ND 2019, Universitat Politècnica de València 
MARKHAM, T. (2003). Project Based Learning, a guide to Standard-focused project based learning for middle and high school teachers. California, USA: Buck Institute for Education.. SANMARTÍN, I (2010). Lipopoliplejos y complejos monomoleculares de ADN como vectores de terapia génica. Tesis Doctoral. Valencia: Universitat de València. $<$ http://roderic.uv.es/handle/10550/23421> [Consulta: 15 de Octubre 2018]

VALERO-GARCÍA, M., GARCÍA ZUBÍA, J. (2011). “Cómo empezar fácil con PBL”. En JENUI 2011, Actas de las Jornadas de Enseñanza Universitaria de la Informática, Sevilla. Disponible en $<$ https://idus.us.es/xmlui/handle/11441/61478> [Consulta: 1 de Octubre de 2018] 